\title{
The costs of target prioritization and the external requirements for using a recall-to-reject strategy in memory exclusion tasks: a meta-analysis
}

\author{
Timm Rosburg ${ }^{1,2}$ (D) Axel Mecklinger ${ }^{1}$
}

Published online: 15 March 2017

(C) Psychonomic Society, Inc. 2017

\begin{abstract}
In event-related potential (ERP) studies, the leftparietal old/new effect is commonly considered as a neural correlate of recollection. In memory exclusion tasks, the effect is usually observed when the targeted information is identified, but it is not necessarily present when studied items are rejected as nontargets. Interestingly, both the presence and the absence of such old/new effects to nontargets have been regarded as indicator for strategic retrieval. We reviewed previous ERP studies using memory exclusion tasks to analyze the reaction time (RT) pattern in such studies, as well as the influence of task difficulty on the occurrence of nontarget retrieval. We identified 44 test conditions, reported in 24 studies, and subjected the behavioral data to a meta-analysis. The RTs to correctly rejected new items were shorter than the RTs to hits, in particular in studies that required differentiating conceptual or perceptual information at test. When the retrieval of target information was prioritized, RTs to nontargets were delayed relative to targets. Without such prioritization, no such RT differences were observed. For test conditions with nontarget retrieval, the retrieval accuracy was poorer compared with test conditions without such retrieval. The findings support previous studies that claimed that nontarget retrieval
\end{abstract}

Electronic supplementary material The online version of this article (doi:10.3758/s13423-017-1256-1) contains supplementary material, which is available to authorized users.

Timm Rosburg

timm.rosburg@upkbs.ch

1 Department of Psychology, Experimental Neuropsychology Unit, Saarland University, Campus A2.4, D-66123 Saarbrücken, Germany

2 Department of Forensic Psychiatry, University Psychiatric Clinics, University Basel, Wilhelm Klein-Str. 27,

CH-4002 Basel, Switzerland becomes more likely when target retrieval is difficult, but the strong overlap in task difficulty between conditions with and without nontarget retrieval indicates that other, partly yet tobe-identified factors contribute to the occurrence of nontarget retrieval as well.

Keywords Episodic memory $\cdot$ Event-related potentials · Recollection $\cdot$ Strategic retrieval $\cdot$ Meta-analysis $\cdot$ Reaction times · Nontarget retrieval $\cdot$ Old/new effects

\section{Introduction}

Strategic retrieval refers to control processes that are engaged to optimize retrieval success but also to minimize retrieval effort (Rosburg, Johansson, \& Mecklinger, 2013). Such control processes have among others been investigated in memory exclusion tasks (Jacoby, 1991). In such memory tasks, items are studied in two (or more) conditions; at the subsequent memory test, items of one study condition are designated as targets, whereas items of the other study condition(s), together with newly presented items, have to be rejected (or "excluded") as nontargets. The rejection of nontargets might be based on the retrieval of nontarget source information in form of a "recall-to-reject strategy" (Clark, 1992). Thus, a rememberer might reject an item as nontarget, because he remembers that this item was presented in the nontarget condition. However, subjects do not necessarily have to retrieve the nontarget source information. They might instead focus on the target information: items are accepted as targets if their recognition is accompanied by the reactivation of sourcespecifying information that matches the target condition, whereas all other (old) items are rejected as nontargets (Herron \& Rugg, 2003b). Such a "target prioritization" has the benefit that the rememberer can constrain his retrieval on a 
single, episodic context. Thus, strategic recollection might be described as the optimization of retrieval, somewhat paradoxically, either by the absence or by the presence of nontarget retrieval ("target prioritization" vs. "recall-to-reject strategy").

In ERP studies, these two forms of strategic recollection in memory exclusion tasks can be evaluated by assessing if nontargets elicited a left-parietal old/new effect (500-800 ms), an ERP effect commonly regarded as a correlate of recollection (Friedman \& Johnson, 2000; Mecklinger \& Jäger, 2009). The presence of a left-parietal old/new effect to nontargets is considered as evidence for a recall-to-reject strategy; its absence is regarded as evidence for target prioritization (Fig. 1) (Rosburg et al., 2013).

From a cognitive development point of view, it has been presumed that target prioritization reflects a stage of functional brain maturation and cannot be utilized until a certain age is reached. Sprondel, Kipp, and Mecklinger (2012) found that, at comparable levels of task difficulty, adult subjects prioritized target information, whereas adolescent subjects retrieved target and nontarget information. The authors argue that this finding "provides strong support for the view that the ability to selectively recollect target information at the expense of nontarget information is generally immature in early adolescence" (Sprondel et al., 2012, p. 11). This view is supported by the finding that the degree of target prioritization increases with higher working memory capacities in adult subjects (Elward \& Wilding, 2010).

The conditions under which adults use one or the other strategy have yet not been fully specified. An influential account suggests that a "recall-to-reject strategy" is used when the retrieval of target information is difficult (Herron \& Rugg, 2003b). In such situations, target information alone is not sufficiently diagnostic for differentiating target and nontargets (Dzulkifli \& Wilding, 2005; Dzulkifli, Herron, \& Wilding, 2006; Wilding, Fraser, \& Herron, 2005; Rosburg et al.,

'Target prioritization'

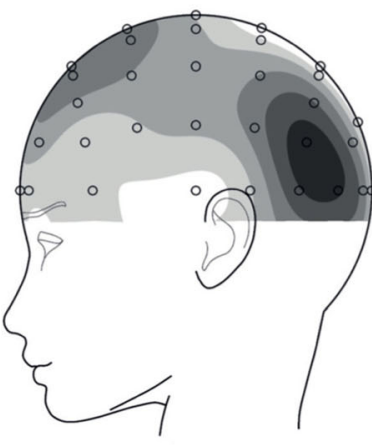

Targets

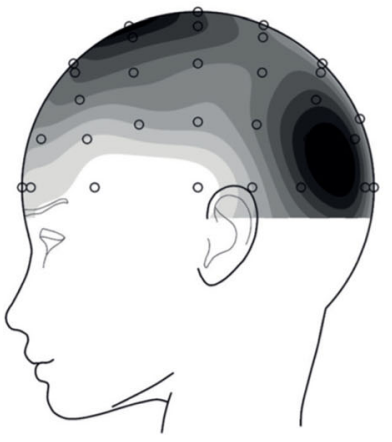

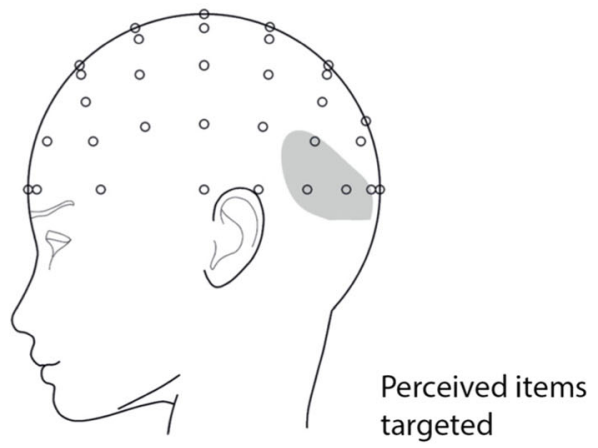

Nontargets

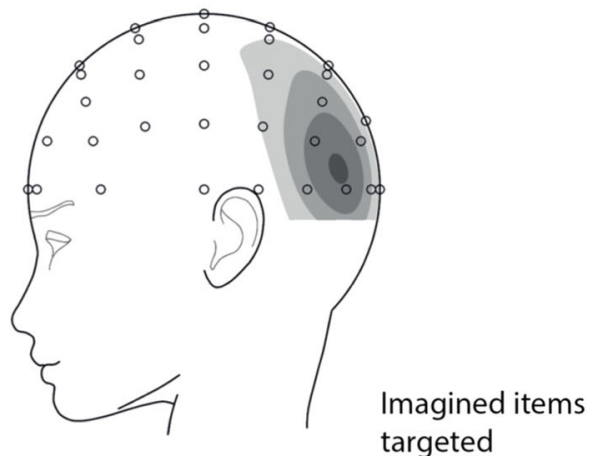

'Recall-to-reject'

$-1 \mu \mathrm{V}$

Fig. 1 The left-parietal old/new effect in memory exclusion tasks for targets and nontargets (exemplary data): The ERPs of correctly identified targets (hits) is more positive going over left-parietal electrode sites than the ERP of correctly rejected new items (CRs). The figure displays this difference potential between old and new items (old/new effect) in its topographical distribution from 500 to $800 \mathrm{~ms}$ for targets and nontargets in the two test conditions (data from Rosburg et al., 2011). Targets elicited a significant left-parietal old/new effect (left) in both conditions, whereas nontargets elicited such an effect in one condition (right bottom) but not in the other (right top). The presence of a left-parietal old/new effect to nontargets is considered as evidence for a recall-to-reject strategy; its absence is regarded as evidence for target prioritization 
2013). However, at least one study reported data that is inconsistent with such target difficulty account (Herron \& Wilding, 2005). The similarity of target and nontarget information, the accessibility of nontarget information, and the class of information to-be-retrieved have been proposed to represent additional factors promoting the retrieval of nontarget information in exclusion tasks (Herron \& Wilding, 2005; Rosburg, Mecklinger, \& Johansson, 2011, Bridger, Sprondel, \& Mecklinger, 2015).

In the current meta-analysis, we reviewed previous ERP studies using memory exclusion tasks to elucidate the external requirements that favor a recall-to-reject strategy. We considered a recall-to-reject strategy as present when nontargets elicited significant parietal old/new effects; we considered target prioritization to be present when nontargets did not elicit significant parietal old/new effects. We favored this approach over the comparison of the size of parietal old/new effects for targets and nontargets, as considered by others (Elward, Evans, \& Wilding, 2013). The major reason for this preference was that, even under recall-to-reject, some attenuation of nontarget old/new effects relative to targets can be anticipated, because ERPs to correctly rejected nontargets comprise responses to nontargets identified as nontargets and nontargets falsely considered as new, i.e., forgotten nontargets (Wilding \& Rugg, 1997). Recollection is absent for the latter and, thus, a parietal old/new effect can be expected to be absent in this subset of trials (Addante, Ranganath, \& Yonelinas, 2012). Because ERPs represent average responses, the inclusion of forgotten trials leads to some attenuation of observed old/new effect to nontargets.

As the main purpose of our study, we sought to test whether a recall-to-reject strategy becomes more likely when the retrieval of target information is difficult (as suggested by the "target difficulty account"; Herron \& Rugg, 2003b). Moreover, we were interested in the reaction time (RT) pattern in memory exclusion tasks and their dependence on nontarget retrieval/target prioritization. In detail, we first compared the RTs to correctly rejected new items $\left(\mathrm{CR}_{\text {new }}\right)$ and correctly identified targets (hits). We hypothesized that the RTs to $\mathrm{CR}_{\text {new }}$ are generally shorter than the RTs to hits, because experiencing the newness of an item is sufficient for its rejection, whereas experiencing oldness needs to be followed by the retrieval of source specifying information before targets can be identified as such. Second, we hypothesized that the RTs to correctly rejected (old) nontargets would be longer than the RTs to targets when target information is prioritized. Following the argumentation of Herron and Rugg (2003b), in this case, old items are rejected as nontargets if target specifying information cannot be retrieved. For nontargets that are recognized as old, such an exclusive memory search requires likely more time than the actual retrieval of this kind of information. This RT delay to nontargets might be considered as cost of prioritization, in particular if no systematic RT delay to nontargets is present when nontarget retrieval occurs. Third, we investigated whether retrieval of nontarget information is dependent on the difficulty for retrieving target information, as suggested by the target-difficulty account (Herron \& Rugg, 2003b). At least two factors might contribute to this target difficulty: (a) the effort to retrieve target information, which might be reflected in the additional time required for identifying targets compared with new items, and (b) the retrieval accuracy as the difficulty to identify the source of an item, which was presumed to be reflected in the discrimination in-

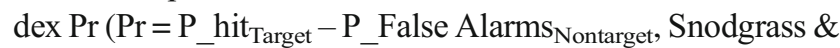
Corwin, 1988). This index is considerably influenced by the rate of forgotten targets. Of note, it does not reflect the discriminability of targets and nontargets, under the precondition that an item was correctly considered as "old" (i.e. was not rejected) but the hit rate corrected by the FA rate to nontargets.

\section{Methods}

\section{Systematic searches and inclusions criteria}

In our meta-analysis, we included only studies that used the memory exclusion task and ERPs. A literature search was conducted in MEDLINE using the following key words ("ERP" or "parietal") and "memory exclusion." In studies detected by this search, the reference lists were checked for additional studies.

Inclusion criteria: Included studies needed to report on the left-parietal ERP old/new effect to targets and nontargets. Studies needed to report the mean RTs ( \pm standard deviations [SD] or standard errors [SE]) to targets, nontargets, and new items, as well as the mean hit rate and rate for correct rejections of nontargets and new items ( $\pm \mathrm{SD} / \mathrm{SE})$. In case of missing values or inconsistent reports, we sought to contact the authors of the studies.

Exclusion criteria: Experiments with pictorial material in which nontarget stimuli were variants of target stimuli were not considered. In some exclusion tasks, cues had to be rejected when they depicted target stimuli with modified aspect ratios (Ranganath \& Paller, 1999) or with modified colors (Ecker, Zimmer, \& Groh-Bordin, 2007). Furthermore, we excluded studies that used a criterial recollection task, because this kind of task is presumed to enforce nontarget retrieval (Bridger \& Mecklinger, 2012) due to the fact that some items are presented in more than one study condition (Gallo et al., 2004). We did not consider data on adolescents (Sprondel, Kipp, \& Mecklinger, 2011) or aged participants (Dywan, Segalowitz, \& Webster, 1998) to avoid taking maturational factors and ageing factors into consideration. Finally, we did not consider ERP studies that did not show left-parietal old/ new effects to targets, because absent left-parietal old/new 
effects to nontargets cannot be interpreted on a functional basis in such cases.

We identified 25 studies that fulfilled our inclusion criteria. These studies often included two test conditions. In all studies (except one), one category of study items was defined as targets and a second category of study items as nontargets. In contrast, in the study by Evans, Wilding, Hibbs, and Herron (2010), there were three kinds of study items and two had to be rejected as nontargets at testing. Data of these two kinds of nontargets were considered as two independent observations for the purpose of the current study.

\section{Data extraction and analysis}

The selected articles were reviewed to extract the following data: sample sizes of the study groups, the conducted study and test conditions, the mean RTs $( \pm$ SD) to targets, nontargets and new items, as well as the mean hit rate and rate for correct rejections of nontargets and new items $( \pm$ SD). Furthermore, it was determined whether nontarget retrieval (as indicated by a significant left-parietal old/new effect) was present or absent.

For the analysis of the RT differences, the mean RTs ( \pm SD) of each study were entered into OpenMeta Analyst (Brown University) and effects sizes of their differences were calculated by using a random effect model. For the analyses, effects either across all conditions or across all conditions that showed target prioritization were expected. To investigate whether retrieval of nontarget information is dependent on the difficulty for retrieving target information, the Pr scores, as well as RT differences between CR of new items and hits were used as dependent variables. Of note, genuine effect sizes were only at hand for the RT differences. For the Pr scores, the individual values of each study were contrasted to the weighted average performance across all studies. Many studies reported hit and false-alarm (FA) rates, but not Pr scores. Therefore, the SD of the Pr scores was estimated on the basis of the pooled SDs of the hit rate and FA rate to nontargets $\left(\sqrt[2]{s^{2} F A+s^{2} \text { hits }}\right.$. For the Pr score, an effect size $>0.0$ reflects a Pr score that is above the average across all studies.

\section{Results}

The presented meta-analysis was based on data of 44 test conditions described in the identified 24 studies. In 24 test conditions, a parietal old/new effect to nontargets was observed, whereas no such nontarget effect was observed in 20 other test conditions (an overview is provided by Supplementary Table S1). The parietal old/new effects for targets and nontargets were contrasted in 18 of the 24 studies ( 35 test conditions): absent nontarget effects were always associated with a larger old/new effect for targets than for nontargets (17 test conditions). In contrast, 8 of 18 conditions with present nontarget effects were associated with significantly larger old/new effects to targets than to nontargets, as well.

In the 24 studies, 588 participants were tested in one or two conditions. In four studies, the gender of the participants $(\mathrm{n}=$ 69) was not specified. Of the remaining 519 participants, 319 were female and 200 were male. Most studies were conducted on students. In consequence, the participants' mean age showed very little variation across studies (range 20-24 years). Given these small variances, age and gender proportion were not considered as covariates for the meta-analysis.

The used encoding tasks and target definitions showed considerable variation across studies. Based on the characteristics of the tasks, we differentiated four kinds of test situations. Following the argumentation of Bridger et al. (2015), conditions in which participants had to discriminate at test between items studied in one or the other encoding task were defined as conceptual memory exclusion tasks (Bridger, 2010; Bridger, Herron, Elward, \& Wilding, 2009; Dzulkifli \& Wilding, 2005; Dzulkifli et al., 2006; Elward et al., 2013; Evans et al., 2010; Herron \& Rugg, 2003b; Herron \& Wilding, 2005; Rosburg et al., 2011; 2013; Rosburg, Johansson, Sprondel, \& Mecklinger, 2014); conditions in which participants had to discriminate at test between items studied in one or the other perceptual condition were defined as perceptual memory exclusion tasks. In the latter type of task, target/nontarget discrimination is based on a perceptual feature, such as the gender of a voice that named an item at study (Bridger et al., 2015; De Chastelaine, Friedman, Cycowicz, 2007; Sprondel et al., 2012; Wilding \& Rugg, 1997; Wilding \& Sharpe, 2004; Wilding et al., 2005). In yet another type of studies, participants had to discriminate two kinds of items that had previously been presented, but at different time points (e.g., in two different study lists). This type of task was defined as temporal memory exclusion task (Bridson, Fraser, Herron, \& Wilding, 2006; Czernochowski, Mecklinger, \& Johansson, 2009; Dywan et al., 1998; Dywan, Segalowitz, Webster, Hendry, \& Harding, 2001; Fraser, Bridson, \& Wilding, 2007; Inaba, Kamishima, \& Ohira, 2007). Finally, in some experiments, study items were presented in two formats, either as words and as pictures, but at test only one format was used for presenting the test cues. For the four conditions included in the analysis, test cues were always words. For the purpose of the current study, these test conditions were conceptualized as format change memory tasks (Herron \& Rugg, 2003a; Johnson \& Rugg, 2006). Of note, format change memory tasks might be considered as a certain kind of conceptual memory task, as the different formats also were associated with different tasks at study. The relative portion of studies showing and not showing a left-parietal old/new effect to nontargets was similar across the task categories (Table 1). In particular, the relative portions did not vary between conceptual and perceptual memory tasks $\left(\chi^{2}=0.385\right.$, n.s. $)$, contrary to the hypothesis of Bridger et al. (2015) who assumed that prioritization of target information is more likely in conceptual memory tasks than in perceptual memory tasks. 
Table 1 Number of studies of each task category showing or not showing a left-parietal old/new effect to nontargets

\begin{tabular}{llll}
\hline Memory test category & \multicolumn{2}{l}{ Nontarget retrieval } \\
\cline { 2 - 4 } & Absent & Present & $\Sigma$ \\
\hline Conceptual & 10 & 12 & 22 \\
Format & 2 & 2 & 4 \\
Perceptual & 3 & 6 & 9 \\
Temporal & 5 & 4 & 9 \\
$\Sigma$ & 20 & 24 & 44 \\
\hline
\end{tabular}

\section{RTs to $\mathbf{C R}_{\text {new }}$ vs. Hits}

We hypothesized that in memory exclusion tasks the RTs to correctly rejected new items are generally shorter than the RTs to correctly identified targets (hits). This hypothesis was verified by the meta-analysis. Compared with hits, participants responded faster to correctly rejected new items (pooled standard mean difference [SMD]: 0.539, 95\% confidence interval [CI]: 0.416-0.663, standard error [SE]: 0.063, $p<0.001$ ). However, statistically significant heterogeneity between the studies was present in the analysis $\left(\tau^{2}=0.062, \mathrm{I}^{2}=37 \%\right.$, $\left.\mathrm{Q}_{42}=66.150, p=0.010\right)$. The analysis of the used memory task showed that the faster RTs to CRs were present in conceptual and perceptual memory tasks (pooled SMD: 0.767, 95\% CI: 0.634-0.899, SE: 0.068, $p<0.001$, and pooled SMD: $0.681,95 \%$ CI: $0.464-0.898$, SE: $0.111, p<0.001$, respectively) but not in format change memory tasks (pooled SMD: $-0.164,95 \%$ CI: -0.502 to 0.173 , SE: 0.172 , n.s.) and temporal memory exclusion tasks (pooled SMD: 0.102, CI: -0.133 to 0.337 , SE: 0.120 , n.s.; Fig. 2). Thus, faster RTs to $\mathrm{CR}_{\text {new }}$ were only found for the two kinds of memory exclusion tasks, in which either conceptual or perceptual information was essential for the target identification and no format change was required.

\section{RTs to nontargets versus hits}

Our second prediction was that in the absence of nontarget retrieval the RTs to correctly rejected (old) nontargets would be longer than the RTs to targets ("costs of prioritization"). This hypothesis was confirmed: In the 20 conditions without nontarget retrieval, the mean RTs for nontargets were significantly longer than for hits (pooled SMD: $-0.248, \mathrm{CI}:-0.393$ 0.102 , SE $0.074, p<0.001$; Fig. 3 ). No statistically significant heterogeneity between the studies was present $\left(\tau^{2}<0.001, \mathrm{I}^{2}\right.$ $<1 \%, \mathrm{Q}_{42}=10.719$, n.s.). Adding TASK as covariate did not yield significant effects. In contrast, in the 24 conditions with nontarget retrieval, the RTs to nontargets were not delayed compared with hits (pooled SMD: -0.072 , CI: -0.196 to 0.052, SE: 0.063, n.s.).

\section{Predictors for nontarget retrieval}

In their account on strategic retrieval, Herron and Rugg (2003b) proposed that retrieval of nontarget information is dependent on the difficulty for retrieving target information. Nontarget retrieval is presumed to be more likely when task difficulty is high. In our meta-analysis, we tested this account by using two operational definitions of task difficulty: the RT difference between $\mathrm{CR}$ to new items and hits targets, as well as the Pr scores for targets.

\section{RT differences between CR of new items and hits targets}

The comparison of the RT effects between conditions that either showed or did not show NT retrieval revealed rather similar effects sizes in the samples, irrespective whether just conceptual and perceptual memory task conditions or all task conditions were analyzed (e.g., combined analysis of conceptual and perceptual memory task conditions: NT retrieval present, $\mathrm{g}=$ 0.748, SE 0.073; NT retrieval absent $g=0.733$, SE 0.091). Thus, similarly slowed-down RTs to hits compared with CR were found in conditions with and without NT retrieval.

\section{Pr scores for targets}

The analysis of the Pr scores revealed a statistically significant heterogeneity $\left(\tau^{2}=0.488, \mathrm{I}^{2}=82 \%, \mathrm{Q}_{43}=235.634, p<0.001\right)$. Inclusion of TASK as moderator variable showed only a tendency for differences between the four kinds of task $(p=0.095)$. Conditions with format tasks showed Pr scores that were significantly above average. (Note that just four conditions used such tasks.) Across all conditions, inclusion of NT retrieval as moderator variable did not show the expected pattern with lower Pr scores for conditions with NT retrieval than for conditions without such retrieval (NT retrieval present, $g=-0.123$, SE 0.163; NT retrieval absent $g=0.063$, SE 0.166). In an additional step, this analysis was restricted to conceptual and perceptual memory tasks because one might consider these two types of tasks as typical exclusion tasks: in this analysis, the expected pattern was observable but was less pronounced than expected ( $p=$ 0.041; Fig. 4). Additional inclusion of the four format change conditions (which, as outlined, might be considered as a certain form of conceptual memory task) would have resulted in a somewhat higher significance $(p=0.020)$.

\section{Discussion}

\section{Main findings}

The main findings of the study can be summarized as follows: We found that the RTs to correctly rejected new items were shorter than the RTs to hits, in particular in studies that required differentiating conceptual or perceptual information at 


\section{Studies}

Bridger (2009) I

Bridger (2009) II

Bridger et al. (2009) I

Bridger et al. (2009) II

Bridger et al. (2015) I

Dzulkifli \& Wilding (2005)

Dzulkifli \& Wilding (2005) II

Dzulkifli et al. (2006) I

Dzulkifli et al. (2006) II

Elward et al. (2013)

Evans et al. (2010) II

Herron \& Rugg (2003b) I

Herron \& Rugg (2003b) II

Herron \& Wilding (2005) ।

Herron \& Wilding (2005) II

Rosburg et al. (2011) I

Rosburg et al. (2011) II

Rosburg et al. (2013) I

Rosburg et al. (2013) II

Rosburg et al. (2014) I

Rosburg et al. (2014) II

Subgroup conceptual (l^2=0\%, P=0.972) $0.767(0.634,0.899)$

Bridger et al. (2015) II

De Chastelaine et al. (2007)

Sprondel et al. (2012) I

Sprondel et al. (2012) II

Wilding \& Rugg (1997)

Wilding \& Sharpe (2004) I

Wilding \& Sharpe (2004) II

Wilding et al. (2005) I

Wilding et al. (2005) II

Subgroup perceptual $\left(\mathbf{I}^{\wedge} \mathbf{2}=\mathbf{0} \%, \mathrm{P}=\mathbf{0 . 6 3 3 )} 0.681(0.464,0.898)\right.$

Bridson et al. (2006) I

Bridson et al. (2006) II

Czernochowski et al. (2009)

Dywan et al. (1998)

Dywan et al. (2001) I

Dywan et al. (2001) II

Fraser et al. (2007) I

Fraser et al. (2007) II

Inaba et al. (2007)

Subgroup temporal $\left(\mathrm{I}^{\wedge} 2=0 \%, \mathrm{P}=\mathbf{0 . 6 2 6}\right)$

Herron \& Rugg (2003a) I

Herron \& Rugg (2003a) II

Johnson \& Rugg (2006) I

Johnson \& Rugg (2006) II

Subgroup format $\left(\mathrm{I}^{\wedge} 2=0 \%, P=0.736\right)$

Overall $\left(\left.\right|^{\wedge} \mathbf{2}=\mathbf{3 7} \%, \mathrm{P}=\mathbf{0 . 0 1 0}\right)$
Estimate (95\% C.I.)

$0.971(0.280,1.661)$

$0.774(0.097,1.451)$

$0.805(0.325,1.285)$

$0.671(0.196,1.146)$

$1.128(0.492,1.764)$

$0.825(0.144,1.505)$

$0.666(-0.005,1.337)$

$0.569(-0.098,1.235)$

$0.517(-0.147,1.181)$

$0.859(0.267,1.450)$

$0.694(-0.019,1.408)$

$0.384(-0.315,1.083)$

$0.429(-0.272,1.130)$

$0.874(0.190,1.558)$

$0.772(0.095,1.449)$

$1.120(0.593,1.647)$

$0.917(0.402,1.432)$

$1.040(0.518,1.562)$

$0.463(-0.033,0.960)$

$0.654(-0.058,1.365)$

$0.643(-0.067,1.354)$

$0.688(0.080,1.296)$

$0.310(-0.387,1.007)$

$1.237(0.561,1.914)$

$1.027(0.367,1.686)$

$0.491(-0.084,1.065)$

$0.783(0.105,1.461)$

$0.687(0.015,1.359)$

$0.572(-0.094,1.239)$

$0.386(-0.274,1.045)$

$0.083(-0.610,0.777)$

$0.146(-0.548,0.840)$

$-0.103(-0.757,0.551)$

$0.383(-0.460,1.227)$

$-0.579(-1.396,0.238)$

$-0.168(-0.885,0.549)$

$0.438(-0.223,1.099)$

$0.081(-0.612,0.774)$

$0.455(-0.189,1.099)$

$0.102(-0.133,0.337)$

$-0.244(-0.900,0.411)$

$-0.373(-1.032,0.286)$

$-0.166(-0.860,0.528)$

$0.158(-0.536,0.852)$

$-0.164(-0.502,0.173)$

$0.538(0.413,0.664)$

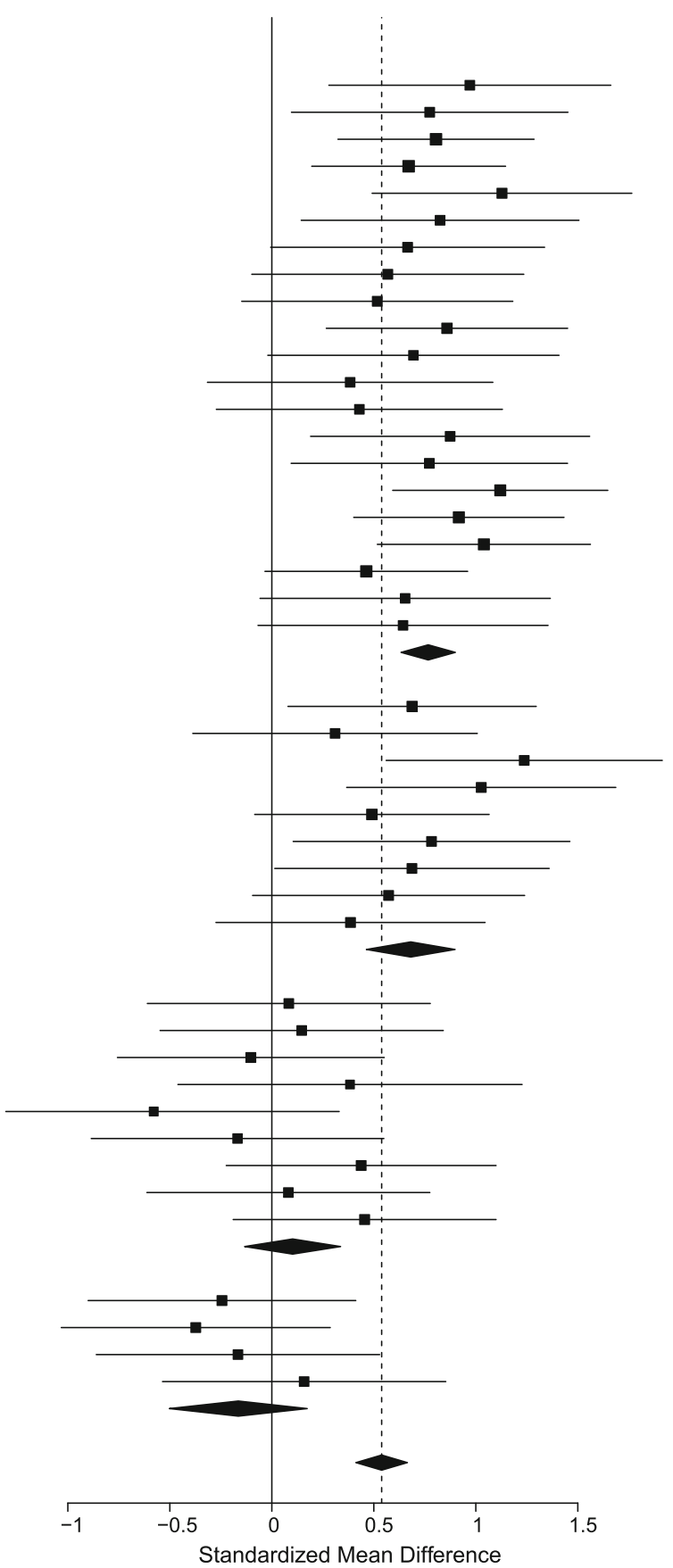

Fig. 2 The standardized mean differences (effect sizes) for RT differences between CR_new and hits, for each of the four memory task categories: Significant differences (with faster RTs for CR_new) were found for perceptual and conceptual memory tasks, but not for temporal and format tasks

test. Furthermore, when the retrieval of target information was prioritized, RTs to nontargets were delayed relative to RTs to targets, whereas no such RT differences were observed when nontarget information was retrieved. With regard to the preconditions for nontarget retrieval, we found some evidence that increasing task difficulty increases the likelihood of nontarget retrieval: For test conditions with nontarget retrieval, the retrieval accuracy for old items was poorer compared with test conditions without such retrieval. In contrast, another presumed marker for task difficulty, the delay between the RTs to correctly rejected new items and the RTs to hits, was not predictive for nontarget retrieval.

\section{RTs to $\mathbf{C R}_{\text {new }}$}

The RTs to $\mathrm{CR}_{\text {new }}$ and to hits were compared to reveal whether there is a systematic difference between the two. We hypothesized that the RTs to $\mathrm{CR}_{\text {new }}$ are generally shorter than the RTs 
Studies

Bridger (2009) I

Bridger (2009) II

Bridger et al. (2009) I

Bridger et al. (2009) II

Bridger et al. (2015) I

Bridger et al. (2015) II

Bridson et al. (2006) I

Bridson et al. (2006) II

Czernochowski et al. (2009)

De Chastelaine et al. (2007)

Dzulkifli et al. (2006) I

Dzulkifli et al. (2006) II

Elward et al. (2013)

Fraser et al. (2007) II

Herron \& Rugg (2003a) I

Herron \& Rugg (2003b) II

Johnson \& Rugg (2006) I

Rosburg et al. (2011) I

Rosburg et al. (2013)

Rosburg et al. (2014) II

Wilding \& Rugg (1997)

Wilding \& Sharpe (2004) I

Wilding \& Sharpe (2004) II

Wilding et al. (2005) I

Subgroup $1\left(\left.\right|^{\wedge} 2=0 \%, P=0.906\right)$

Dywan et al. (1998)

Dywan et al. (2001) I

Dywan et al. (2001) II

Dzulkifli \& Wilding (2005) ।

Dzulkifli \& Wilding (2005) II

Evans et al. (2010) I

Evans et al. (2010) II

Fraser et al. (2007) I

Herron \& Rugg (2003a) II

Herron \& Rugg (2003b) I

Herron \& Wilding (2005) ।

Herron \& Wilding (2005) II

Inaba et al. (2007)

Johnson \& Rugg (2006) II

Rosburg et al. (2011) II

Rosburg et al. (2013) II

Rosburg et al. (2014) I

Sprondel et al. (2012) I

Sprondel et al. (2012) II

Wilding et al. (2005) II

Subgroup $0\left(\left.\right|^{\wedge} \mathbf{2}=0 \%, P=0.933\right)-0.248(-0.393,-0.102)$

Overall $\left(\left.\right|^{\wedge} 2=0 \%, P=0.955\right) \quad-0.146(-0.240,-0.051)$
Estimate (95\% C.I.)

$0.163(-0.492,0.817)$

$-0.137(-0.791,0.517)$

$-0.170(-0.633,0.292)$

$-0.147(-0.609,0.316)$

$-0.152(-0.743,0.440)$

$-0.048(-0.639,0.544)$

$0.086(-0.607,0.780)$

$0.392(-0.308,1.091)$

$-0.311(-0.968,0.346)$

$-0.445(-1.146,0.257)$

$-0.098(-0.752,0.555)$

$-0.163(-0.817,0.492)$

$-0.199(-0.766,0.369)$

$0.237(-0.458,0.932)$

$-0.101(-0.755,0.552)$

$0.086(-0.607,0.779)$

$-0.909(-1.637,-0.181)$

$0.163(-0.328,0.654)$

$0.303(-0.189,0.796)$

$0.046(-0.647,0.739)$

$0.018(-0.548,0.584)$

$-0.221(-0.876,0.434)$

$-0.215(-0.870,0.440)$

$-0.249(-0.905,0.407)$

$-0.153(-0.990,0.684)$

$0.332(-0.474,1.137)$

$-0.102(-0.818,0.614)$

$-0.253(-0.908,0.403)$

$0.000(-0.653,0.653)$

$0.119(-0.574,0.813)$

$0.042(-0.651,0.735)$

$-0.093(-0.747,0.560)$

$-0.737(-1.413,-0.062)$

$-0.160(-0.854,0.535)$

$-0.201(-0.856,0.454)$

$-0.198(-0.853,0.457)$

$-0.026(-0.662,0.610)$

$-0.302(-0.999,0.395)$

$-0.419(-0.914,0.077)$

$-0.487(-0.984,0.010)$

$-0.646(-1.357,0.064)$

$-0.212(-0.834,0.409)$

$-0.426(-1.053,0.200)$

$-0.491(-1.154,0.172)$

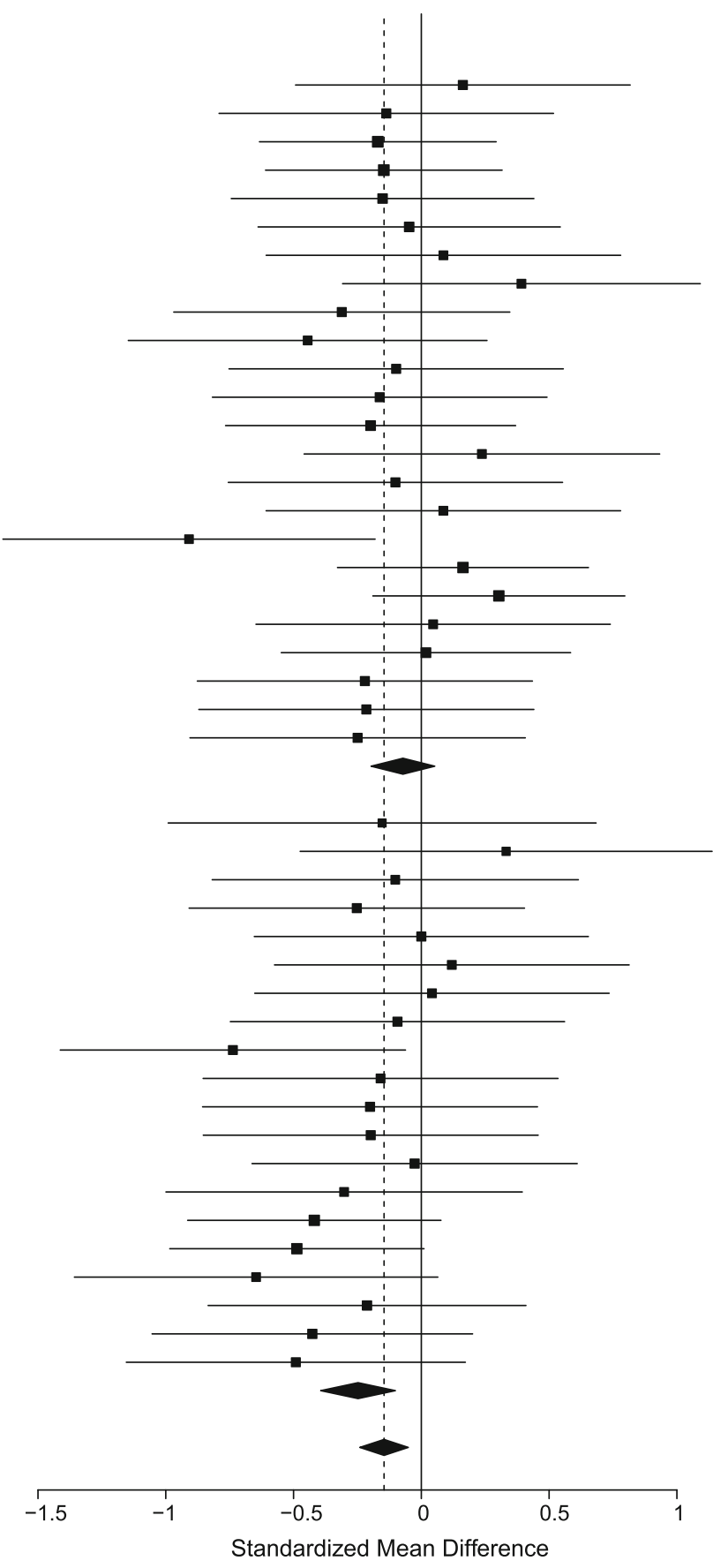

memory exclusion tasks: Conditions without nontarget retrieval showed delayed responses to nontargets compared with the RTs to targets, whereas conditions with nontarget retrieval did on average not show such delay

low diagnostic value of newness when performing the latter two kinds of tasks. In format change memory tasks, the newness of an item is not sufficient for rejecting it as "new," because a feeling of newness also might occur when an item is presented at test in a different format than it has been presented at study (Stenberg, Johansson, \& Rosén, 2006). This might encourage subjects to focus to greater extent on matches between presented test items and stored target information. In 


$\begin{array}{lc}\text { Studies } & \text { Estimate }(95 \% \text { C.I.) } \\ \text { Bridger (2010) I } & 0.538(-0.127,1.203) \\ \text { Bridger (2010) II } & 0.972(0.281,1.662) \\ \text { Bridger et al. (2009) I } & 0.670(0.195,1.145) \\ \text { Bridger et al. (2009) II } & 0.983(0.494,1.472) \\ \text { Bridger et al. (2015) I } & 0.044(-0.548,0.635) \\ \text { Bridger et al. (2015) II } & -0.081(-0.672,0.510) \\ \text { De Chastelaine et al. (2007) } & -0.265(-0.961,0.431) \\ \text { Dzulkifli et al. (2006) I } & -0.761(-1.438,-0.085) \\ \text { Dzulkifli et al. (2006) II } & -0.390(-1.049,0.270) \\ \text { Elward et al. (2013) } & -0.081(-0.647,0.485) \\ \text { Herron \& Rugg (2003b) II } & -0.635(-1.346,0.075) \\ \text { Rosburg et al. (2011) I } & 0.544(0.045,1.043) \\ \text { Rosburg et al. (2013) I } & -1.582(-2.143,-1.020) \\ \text { Rosburg et al. (2014) II } & -0.759(-1.476,-0.041) \\ \text { Wilding \& Rugg (1997) } & -1.575(-2.223,-0.927) \\ \text { Wilding \& Sharpe (2004) I } & -0.637(-1.307,0.032) \\ \text { Wilding \& Sharpe (2004) II } & -0.390(-1.049,0.270) \\ \text { Wilding et al. (2005) I } & -1.132(-1.836,-0.429) \\ \text { Subgroup 1 (I^2=85\% , P=0.000) } & -0.239(-0.600,0.123) \\ & \\ \text { Dzulkifli \& Wilding (2005) I } & 0.848(0.166,1.530) \\ \text { Dzulkifli \& Wilding (2005) II } & 0.910(0.223,1.596) \\ \text { Evans et al. (2010) I } & -0.080(-0.773,0.613) \\ \text { Evans et al. (2010) II } & -0.389(-1.088,0.311) \\ \text { Herron \& Rugg (2003b) I } & 0.105(-0.589,0.798) \\ \text { Herron \& Wilding (2005) I } & -0.576(-1.242,0.091) \\ \text { Herron \& Wilding (2005) II } & -0.204(-0.859,0.451) \\ \text { Rosburg et al. (2011) II } & 0.982(0.463,1.500) \\ \text { Rosburg et al. (2013) II } & 0.606(0.105,1.108) \\ \text { Rosburg et al. (2014) I } & 0.352(-0.347,1.050) \\ \text { Sprondel et al. (2012) I } & -0.019(-0.638,0.601) \\ \text { Sprondel et al. (2012) II } & 0.664(0.027,1.300) \\ \text { Wilding et al. (2005) II } & 0.167(-0.487,0.822) \\ \text { Subgroup 0 (I^2=60\% , P=0.003) } & 0.276(-0.001,0.553) \\ & -0.027(-0.280,0.226)\end{array}$

Fig. 4 The target Pr across perceptual and conceptual memory tasks, for conditions showing nontarget retrieval (top, "subgroup 1") and not showing nontarget retrieval (bottom, "subgroup 0"). A low target $\mathrm{Pr}$

temporal memory exclusion tasks, other factors might come into play, such as a stronger reliance on memory strength, either for identifying repeated items as targets or for rejecting them as nontargets (Hintzman, 2005; Greve, Doidge, Evans, \& Wilding, 2010).

The finding indicates that, even though the task across the defined four kinds of memory exclusion tasks is principally the same, the demands apparently vary between them. A speeded response to $\mathrm{CR}_{\text {new }}$, compared with hits, is only found for conceptual and perceptual memory exclusion tasks. This RT pattern varies from recognitions tasks in which targets are usually identified faster than lures and not the other way around (Johansson, Stenberg, Lindgren, \& Rosén, 2002; Johnson \& Rugg, 2006). The differential pattern in RTs (between $\mathrm{CR}_{\text {new }}$ and hits) in the four kinds of exclusion tasks also implies that this task differentiation is potentially of relevance when considering the requirements for nontarget retrieval, as the main objective of the current study.

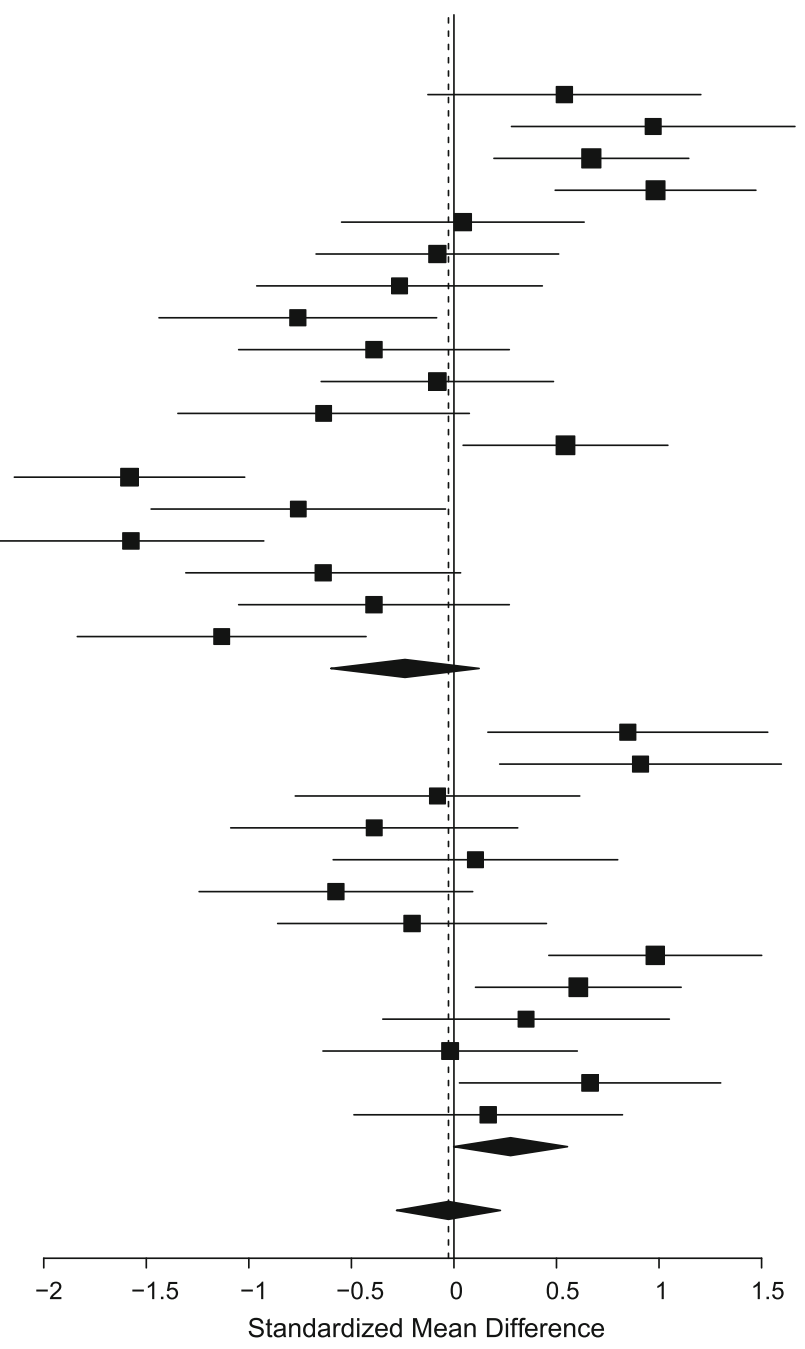

was predictive for the occurrence of nontarget retrieval but just for these two kinds of memory exclusion tasks

\section{RTs to nontargets}

We hypothesized that the RTs to correctly rejected (old) nontargets would be longer than the RTs to targets when target information is prioritized. This hypothesis was confirmed. The delay for nontargets when target information prioritized is in accordance with the account of Herron and Rugg (2003b) who argued that in such cases old items are rejected as nontargets if target specifying information cannot be retrieved. As outlined, such an exclusive memory search likely requires more time than the actual retrieval of this kind of information. Such RT delays to nontargets might be considered as costs of prioritization. Thus, the advantage of prioritization, namely focusing on just one study context (rather than on two or more), comes at a price, namely that additional time is required before studied items can be rejected as nontargets. Importantly, such a systematic delay for nontarget rejection was not observed when nontarget retrieval occurred. Of note, 
RT delays to nontargets (compared with targets) also might occur when nontarget information is retrieved, but such delays are not offset by faster RTs to nontargets in other test conditions. Such faster RTs to nontargets might particularly occur when nontarget information is more easily accessible than target information (Rosburg et al., 2011).

The finding indicates that target prioritization might optimize retrieval effort and retrieval success for target information but also represents a tradeoff with the speed for nontarget identification. This then might imply that response deadline procedures (thus, enforcing faster responses) or experienced time pressure might encourage participants to use a recall-toreject strategy instead of target prioritization.

\section{Task difficulty and nontarget retrieval}

For main objective of the current study, we sought to investigate whether retrieval of nontarget information is dependent on the difficulty for retrieving target information, as suggested by the account of Herron and Rugg (2003b). This difficulty was operationalized by two variables: (a) the RT difference between $\mathrm{CR}_{\text {new }}$ and hits as effort for retrieving source specifying target information; (b) the discrimination index $\mathrm{Pr}$ as measure of retrieval accuracy. Previous evidence for this account was based on the studies of Herron and Rugg (2003b), Dzulkifli and Wilding, 2005, Wilding et al. (2005), and Dzulkifli et al. (2006) in which each two retrieval conditions with varying difficulty were used for the memory exclusion task. In these studies, nontarget retrieval was only present in the more difficult test condition.

Of the two currently analyzed markers for retrieval difficulty, the discrimination index Pr was predictive for nontarget retrieval but only for perceptual and conceptual memory exclusion tasks. Even for these two kinds of tasks, there is some considerable overlap between conditions that showed or that did not show nontarget retrieval (Fig. 4). Thus, the findings underline that retrieval difficulty for target information has some influence on whether nontarget information is retrieved, but this is apparently not the only determinant, as evidenced by some remarkable exceptions from the rule: In two studies by Bridger et al. (2009) and Bridger (2010), nontarget information was retrieved even though retrieval accuracy for target information was at a high level (note that the old/new effects of the first study are reported in Bridger, 2010). In contrast, in the study by Herron and Wilding (2005), nontarget retrieval was absent even though it was attempted to increase the retrieval difficulty by introducing a delay between study and test for target information.

Herron and Wilding (2005) argued, in an elaboration of their previous account, that target information might be prioritized as long as targets have been manipulated in a sufficiently elaborative manner, even when retrieval accuracy decreases. In such cases, target information would remain sufficiently distinct and allow prioritization. However, based on this post-hoc argumentation, it is difficult to explain why nontarget retrieval did occur in the studies by Bridger et al. (2009) and Bridger (2010) where the items were encoded in relatively distinct conditions and, in all likelihood, in a sufficiently elaborative manner, as indicated by the high retrieval accuracy. Moreover, previous studies revealed no evidence that nontarget retrieval becomes more likely when target and nontarget information is similar (Wilding et al., 2005; Evans et al., 2010; Rosburg et al., 2011).

We have previously extended the target difficulty account of Herron and Rugg (2003b) and proposed that the accessibility of nontarget information possibly represents another important factor for nontarget retrieval (Rosburg et al., 2011). We argued that, if nontarget information is easily accessible, it might be retrieved incidentally. This argumentation was based on the observation that the degree of nontarget retrieval (as reflected in the leftparietal old/new effect to nontargets) was larger the better this information was retrieved when it was targeted (Rosburg et al., 2011). Such incidental nontarget retrieval may account for the presence of a left-parietal old/new effect to nontargets in test conditions with high retrieval accuracies for the target information (Czernochowski, Mecklinger, \& Johansson, 2009). Conversely, for other test situations, it is conceivable that participants abort retrieval attempts for nontarget information if this nontarget information is difficult to access (or at least noticeably more difficult to access than the target information).

In general, the provision of evidence for the accounts of Herron and Rugg (2003b) and Rosburg et al. (2011) is complicated by the fact that the retrieval difficulty for target information and the accessibility of nontarget information can only be assessed on the basis of retrieval accuracy measures. Such measures are, however, likely influenced by the presence or absence of nontarget retrieval. Thus, retrieval accuracy for target (and nontarget) information might be considered as the product of the accessibility of target and nontarget information, the distinctiveness of the two kinds of information, and used strategies. Some illustrative examples how the accessibility of one source information influences the retrieval accuracy of a second source are provided by Leynes, Cairns, and Crawford (2005) and Leynes (2012): In these source memory experiments, the retrieval accuracy for identically encoded items was better when items of the second source were better remembered, as well. In this respect, one has to be aware that the estimation of the retrieval difficulty on the basis of accuracy measures alone underlies a certain degree of ambiguity.

Furthermore, in the current analysis we opted to differentiate test conditions on the basis of whether a left-parietal old/new effect to nontargets was present or absent. Alternatively, one might consider differences in the amplitude of the parietal old/ new effects for nontargets and targets as evidence for target prioritization (Elward et al., 2013). However, as outlined in the introduction, even under recall-to-reject some attenuation of nontarget old/new effects relative to targets can be anticipated, because ERPs to correctly rejected nontargets comprise responses to nontargets identified as nontargets and nontargets that were 
forgotten and, by this, falsely considered as new items. We found that across studies, absent parietal old/new effects to nontargets were always associated with larger parietal old/new effects for targets than for nontargets (when reported). Thus, from our point of view, absent parietal old/new effects to nontargets more reliably indicate target prioritization than simply differences in the amplitude of the parietal old/new effects for nontargets and targets. In reverse, the presence of significant parietal old/new effects to nontargets indicates that nontarget information was recollected in considerable extent.

For the sake of completeness, it should be mentioned that, in yet another approach, Bridger et al. (2015) proposed that differences in the amplitudes of nontarget old/new effects across test conditions in the absence of corresponding differences of target effects indicated target prioritization (for the condition with the smaller nontarget old/new effect). We principally agree with this notion, but such a definition of target prioritization presupposes an assessment of relative differences of nontarget effects across test conditions. By this, it would not allow classifying individual memory exclusion task conditions as conditions in which target prioritization or a recall-to-reject strategy took place. Moreover, related to this issue, this approach has its limitations when the two considered test conditions show target old/new effects, but nontarget effects are widely absent in both (Herron \& Wilding, 2005). It is important to stress that all three approaches (presence/absence of old/new effects to nontargets; comparison of old/new effects to targets and nontargets; comparison old/new effects to nontargets across test conditions) represent operational definitions for presumably underlying retrieval strategies and do not allow their definite determination.

Finally, it is important to stress that, within a given test condition, the preferred strategy (recall-to-reject vs. target prioritization) might vary across participants. Substantially larger parietal old/new effects for targets than for nontargets might occur in the presence of parietal old/new effects to nontargets, because some of the participants prioritized target information and others relied on a recall-to-reject strategy. This suggests that some mechanisms of the strategic retrieval might better be addressed in individual studies in which retrieval difficulty (for target or nontarget information) is systematically varied. Such studies might be better suited than the current metaanalysis for testing alternative accounts on nontarget retrieval, such as that the accessibility of nontarget information possibly represents another important factor for nontarget retrieval (Rosburg et al., 2011). High accessibility of nontarget information could trigger involuntary, bottom-up driven recollection and could, in principle, deter the rememberer from target prioritization. Previous studies on strategic retrieval have varied the accessibility of target information (Herron \& Rugg, 2003 b) but not of nontarget information. In a recent own study, we observed larger left-parietal old/new effects to nontargets than to targets (Rosburg et al., 2013), which is an unusual pattern and which we explained by the high accessibility of nontarget information, as compared to the low accessibility of the target information.

\section{Study limitations}

Because meta-analyses are based on group data, the modulation of the nontarget old/new effects by individual withinstudy factors, such as the individual response speed relative to the sample's average response speed, could not be considered in our study. One such individual factor that has been discussed as modulator of nontarget retrieval is the working memory capacity (WMC). It has been reported that the degree of target prioritization increases with higher WMCs in adults (Elward \& Wilding, 2010; Elward et al., 2013). However, inspection of the magnitude of the parietal old/new effects in the samples with low and high WMC in the aforementioned study suggests that the magnitude of the left-parietal old/new effect to nontargets was rather similar, whereas the leftparietal old/new effect to targets varied between groups (see also the correlation pattern between the WMC and parietal old/new effect to targets and nontargets, Elward \& Wilding, 2010; Fig. 3). Based on this pattern, it appears that lower WMC limits the magnitude of the parietal old/new effect to targets rather than the reliance on nontarget retrieval.

The differential modulation of the parietal old/new effect to targets and nontargets by WMC might indicate that, at least in some instances, retrieval for target information is top-down controlled (WMC dependent), whereas retrieval of nontarget information occurs incidentally and is bottom-up modulated (WMC independent). In any case, it appears that nontarget retrieval is not used as compensatory mechanism for the difficulty to retrieve target information, because the left-parietal old/new effects to targets correlate positively with the parietal old/new effects to nontargets, as previously shown: We demonstrated that participants showed less evidence for nontarget retrieval when they had small left-parietal old/new effect to targets (Rosburg et al., 2013). This is contrary to what might be derived from the hypothesis of Herron and Rugg (2003b) but supports the notion that the accessibility of nontarget information is relevant for nontarget retrieval.

\section{Conclusions}

The likelihood for retrieving nontarget information increases with task difficulty, as reflected in the target Pr, supporting the account of Herron and Rugg (2003b) for nontarget retrieval. However, the link between task difficulty and nontarget retrieval was less pronounced than expected, because it was restricted to perceptual and conceptual memory exclusion tasks and there was a large overlap in task difficulty between conditions with and without nontarget retrieval. The influence 
of other potential factors (either more task-related factors, such as the accessibility of nontarget information, or more individual-related factors, such as the WMC) requires further research.

Acknowledgements T.R. was financially supported by the German Research Foundation (DFG Ro 3533/1-1). The authors greatly appreciated the comments of Ed Wilding and two anonymous reviewers on a previous version of this meta-analysis. They confirm that there are no known conflicts of interest associated with this publication, and there has been no significant financial support for this work that could have influenced its outcome.

\section{References}

Addante, R. J., Ranganath, C., \& Yonelinas, A. P. (2012). Examining ERP correlates of recognition memory: Evidence of accurate source recognition without recollection. NeuroImage, 62(1), 439-450.

Bridger EK (2010) Electrophysiological correlates of individual differences in strategic retrieval processing. PhD thesis. Cardiff University.

Bridger, E. K., Herron, J. E., Elward, R. L., \& Wilding, E. L. (2009). Neural correlates of individual differences in strategic retrieval processing. Journal of Experimental Psychology: Learning, Memory, and Cognition, 35(5), 1175-1186.

Bridger, E. K., \& Mecklinger, A. (2012). Electrophysiologically dissociating episodic preretrieval processing. Journal of Cognitive Neuroscience, 24(6), 1476-1491.

Bridger, E. K., Sprondel, V., \& Mecklinger, A. (2015). Control over recollection varies with context-type: ERP evidence from the exclusion task. Cognitive Neuroscience, 6(1), 31-38.

Bridson, N. C., Fraser, C. S., Herron, J. E., \& Wilding, E. L. (2006). Electrophysiological correlates of familiarity in recognition memory and exclusion tasks. Brain Research, 1114(1), 149-160.

Clark, S. E. (1992). Word frequency effects in associative and item recognition. Memory and Cognition, 20(3), 231-243.

Czernochowski, D., Mecklinger, A., \& Johansson, M. (2009). Agerelated changes in the control of episodic retrieval: An ERP study of recognition memory in children and adults. Developmental Science, 12, 1026-1040.

De Chastelaine, M., Friedman, D., \& Cycowicz, Y. M. (2007). The development of control processes supporting source memory discrimination as revealed by event-related potentials. Journal of Cognitive Neuroscience, 19(8), 1286-1301.

Dywan, J., Segalowitz, S. J., Webster, L., Hendry, K., \& Harding, J. (2001). Event-related potential evidence for age-related differences in attentional allocation during a source monitoring task. Developmental Neuropsychology, 19(1), 99-120.

Dywan, J., Segalowitz, S. J., \& Webster, L. (1998). Source monitoring: ERP evidence for greater reactivity to nontarget information in older adults. Brain and Cognition, 36(3), 390-430.

Dzulkifli, M. A., Herron, J. E., \& Wilding, E. L. (2006). Memory retrieval processing: Neural indices of processes supporting episodic retrieval. Neuropsychologia, 44(7), 1120-1130.

Dzulkifli, M. A., \& Wilding, E. L. (2005). Electrophysiological indices of strategic episodic retrieval processing. Neuropsychologia, 43(8), $1152-1162$

Ecker, U. K. H., Zimmer, H. D., \& Groh-Bordin, C. (2007). The influence of object and background color manipulations on the electrophysiological indices of recognition memory. Brain Research, 1185, 221230
Elward, R. L., Evans, L. H., \& Wilding, E. L. (2013). The role of working memory capacity in the control of recollection. Cortex, 49, 14521462.

Elward, R. L., \& Wilding, E. L. (2010). Working memory capacity is related to variations in the magnitude of an electrophysiological marker of recollection. Brain Research, 1342, 55-62.

Evans, L. H., Wilding, E. L., Hibbs, C. S., \& Herron, J. E. (2010). An electrophysiological study of boundary conditions for control of recollection in the exclusion task. Brain Research, 1324, 43-53.

Fraser, C. S., Bridson, N. C., \& Wilding, E. L. (2007). Controlled retrieval processing in recognition memory exclusion tasks. Brain Research, $1150,131-142$.

Friedman, D., \& Johnson, R. (2000). Event-related potential (ERP) studies of memory encoding and retrieval: A selective review. Microscopy Research and Technique, 51(1), 6-28.

Gallo, D. A., Weiss, J. A., \& Schacter, D. L. (2004). Reducing false recognition with criterial recollection tests: Distinctiveness heuristic versus criterion shifts. Journal of Memory and Language, 51(3), 473-493.

Greve, A., Doidge, A. N., Evans, C. J., \& Wilding, E. L. (2010). Functional neuroanatomy supporting judgments of when events occurred. The Journal of Neuroscience, 30(20), 7099-7104.

Herron, J. E., \& Rugg, M. D. (2003a). Retrieval orientation and the control of recollection. Journal of Cognitive Neuroscience, 15 , 843-854.

Herron, J. E., \& Rugg, M. D. (2003b). Strategic influences on recollection in the exclusion task: Electrophysiological evidence. Psychonomic Bulletin and Review, 10(3), 703-710.

Herron, J. E., \& Wilding, E. L. (2005). An electrophysiological investigation of factors facilitating strategic recollection. Journal of Cognitive Neuroscience, 17(5), 777-787.

Hintzman, D. L. (2005). Memory strength and recency judgments. Psychonomic Bulletin and Review, 12(5), 858-864.

Inaba, M., Kamishima, K., \& Ohira, H. (2007). An electrophysiological comparison of recollection for emotional words using an exclusion recognition paradigm. Brain Research, 1133, 100-109.

Jacoby, L. L. (1991). A process dissociation framework: Separating automatic from intentional uses of memory. Journal of Memory and Language, 30, 513-541.

Johansson, M., Stenberg, G., Lindgren, M., \& Rosén, I. (2002). Memory for perceived and imagined pictures: An event-related potential study. Neuropsychologia, 40(7), 986-1002.

Johnson, J. D., \& Rugg, M. D. (2006). Modulation of the electrophysiological correlates of retrieval cue processing by the specificity of task demands. Brain Research, 1071(1), 153-164.

Leynes, P. A. (2012). Event-related potential (ERP) evidence for sourcemonitoring based on the absence of information. International Journal of Psychophysiology, 84, 284-295.

Leynes, P. A., Cairns, A., \& Crawford, J. T. (2005). Event-related potentials indicate that reality monitoring differs from external source monitoring. American Journal of Psychology, 118, 497-524.

Mecklinger A, Jäger T (2009) Episodic memory storage and retrieval: Insights from electrophysiological measures. In: Rösler F, Ranganath C, Röder B, Kluwe RH (eds) Neuroimaging and psychological theories of human memory. Oxford University Press, pp 357-382.

Ranganath, C., \& Paller, K. A. (1999). Frontal brain potentials during recognition are modulated by requirements to retrieve perceptual detail. Neuron, 22(3), 605-613.

Rosburg, T., Johansson, M., Sprondel, V., \& Mecklinger, A. (2014). Retrieving self-vocalized information: An event-related potential (ERP) study on the effect of retrieval orientation. Brain and Cognition, 92C, 123-132.

Rosburg, T., Johansson, M., \& Mecklinger, A. (2013). Strategic retrieval and retrieval orientation in reality monitoring studied by eventrelated potentials (ERPs). Neuropsychologia, 51(3), 557-571. 
Rosburg, T., Mecklinger, A., \& Johansson, M. (2011). Strategic retrieval in a reality monitoring task. Neuropsychologia, 49(10), 2957-2969.

Snodgrass, J. G., \& Corwin, J. (1988). Pragmatics of measuring recognition memory: Applications to dementia and amnesia. Journal of Experimental Psychology: General, 117(1), 34-50.

Sprondel, V., Kipp, K. H., \& Mecklinger, A. (2011). Developmental changes in item and source memory: Evidence from an ERP recognition memory study with children, adolescents, and adults. Child Development, 82(6), 1638-1953.

Sprondel, V., Kipp, K. H., \& Mecklinger, A. (2012). Electrophysiological evidence for late maturation of strategic episodic retrieval processes. Developmental Science, 15(3), 330-344.
Stenberg, G., Johansson, M., \& Rosén, I. (2006). Conceptual and perceptual memory: Retrieval orientations reflected in event-related potentials. Acta Psychologica, 122, 174-205.

Wilding, E. L., Fraser, C. S., \& Herron, J. E. (2005). Indexing strategic retrieval of colour information with event-related potentials. Brain Research. Cognitive Brain Research, 25(1), 19-32.

Wilding, E. L., \& Rugg, M. D. (1997). An event-related potential study of memory for words spoken aloud or heard. Neuropsychologia, 35(9), $1185-1195$.

Wilding, E., \& Sharpe, H. (2004). The influence of response-time demands on electrophysiological correlates of successful episodic retrieval. Cognitive Brain Research, 18(2), 185-195. 